\title{
Effect of Subjective Well-being and Quality of Work Life on Job Commitment of Hospital Staff
}

\section{ART ICLE INF O}

\section{Article Type}

Descriptive Study

\section{Authors}

Mansourlakouraj A.*1 $M S C$

Abdollahi D. ${ }^{2} P h D$

Rasouli I. ${ }^{3} P h D$

How to cite this article

Mansourlakouraj A, Abdollahi D, Rasouli I. Effect of Subjective Wellbeing and Quality of Work Life on Job Commitment of Hospital Staff. Iranian Journal of War \& Public Health. 2019;11(3):169-174.
${ }^{1}$ Management Department, Ardabil Branch, Applied University of Industrial Management, Ardabil, Iran ${ }^{2}$ Social Sciences Department, Humanities Faculty, Farhangian University, Ardabil, Iran

${ }^{3}$ Management Department, Humanities Faculty, Ardabil Branch, Islamic Azad University, Ardabil, Iran

\section{*Correspondence}

Address: Isar Hospital, Shahid Avazzadeh Square, Ataee Street, Ardabil. Postal Code: 5619636733

Phone: +98 (45) 33511010

Fax: +98 (45) 33511508

amin.mansour1300@gmail.com

\section{Article History}

Received: December 29, 2018

Accepted: May 7, 2019

ePublished: July 21, 2019

\section{A B S T R A C T}

Aims Job commitment is one of the characteristics of a positive relationship between an organization and its employees that makes it effective and conducive the performance of the organization. Regarding the importance of this issue, the present study was conducted with the aim of investigating the effect of subjective well-being and quality of work life on job commitment of staff of Isar Hospital, Ardabil.

Instrument \& Methods In the present descriptive correlational study conducted in 2018, all the staff of Isar Hospital were selected and examined. The instruments, including Blavo Career Commitment Scale, standard questionnaire of quality of work life of Walton, and subjective well-being scale of Keyes and Magyar-Moe were used to collect the required information. The data were analyzed by SPSS 23 software, using Pearson correlation coefficient and multivariate regression analysis.

Findings There was a positive and significant correlation between subjective well-being $(p<0.01 ; r=0.78)$ and quality of work life $(p<0.01 ; r=0.42)$. Subjective well-being alone expressed $60.8 \%$ of the variance in job commitment of hospital staff and by adding quality of work life, the value reached $62.0 \%(\mathrm{p}<0.01)$.

Conclusion The high subjective well-being and quality of work life increase job commitment in hospital staff.

Keywords Job Commitment; Subjective Wellbeing; Quality of Work Life; Staff; Hospital

\section{I T A T I O N L I N K S}

[1] The relationship between job commitment and ... [2] The study of job commitment and trust within the organization ... [3] The effects of ethical leadership on manager job satisfaction ... [4] The relationship between work ethic, job commitment ... [5] The relationship among employees' empowerment, organizational commitment ... [6] The causal relationship between subjective well-being, quality of life ... [7] Employee wellbeing and the HRM-organizational performance ... [8] Relationship between components of quality of work life ... [9] An investigation of the relationship between quality of work Life and middle ... [10] Relationship between quality of work life and occupational stress in ... [11] The influence of quality of work life on employee job satisfaction ... [12] Theoretical and practical research in the humanities ... [13] Nurses' career commitment and job performance ... [14] The measurement and utility of adult subjective ... [15] Proposed model of subjective well-being and happiness ... [16] Relationship between quality of work life and organizational ... [17] IQuality of working Life: What ... [18] Assessing the quality of work life, productivity of nurses and ... [19] Relationship between the meaning of life and optimism with ... [20] The relationship of work-family conflict and spillover to affective well-being of ... [21] The role of lifestyle, psychological capital and self-care behaviors in predicting ... [22] Health psychology: Biopsychosocial ... [23] Well-being and employee health-How employees' well-Being scores interact ... [24] When necessity is the mother of invention: Mood and ... [25] Is sexual well-being part of subjective well-being? An empirical ... [26] Exploring perspectives of medical staff on hospital's effects on ... [27] Modeling of the relationship between the quality of work life and organizational commitment by means of job satisfaction ... [28] Quality of work life of nurses working at tertiary health care ... [29] Predictors of quality of work life and job performance in ... 
سازمان و احساس مفيدبودن براى فرد مى شود كه اين خود

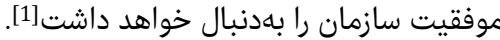

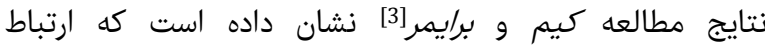

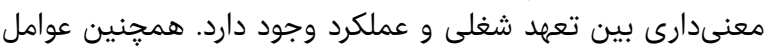

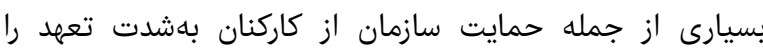

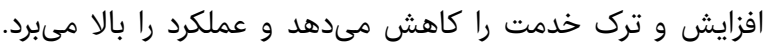

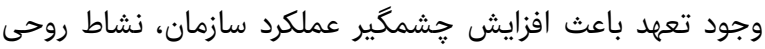

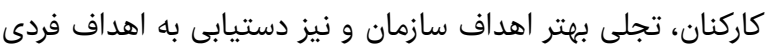

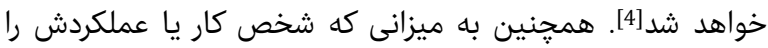

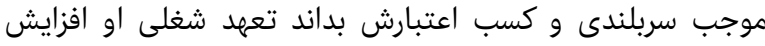

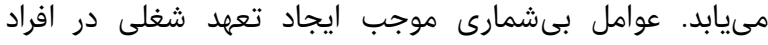

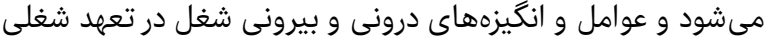

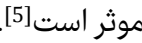

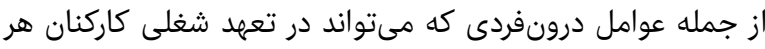

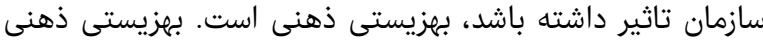

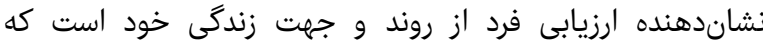

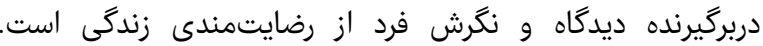

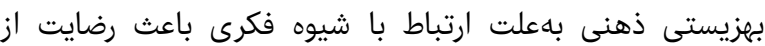

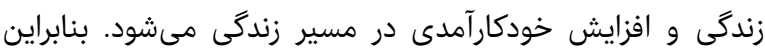

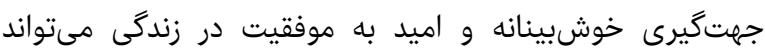

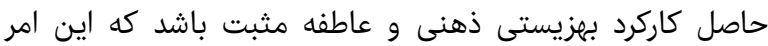

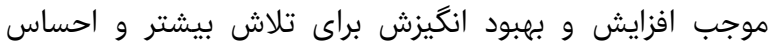

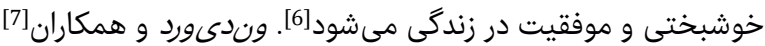

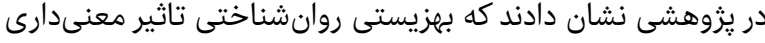

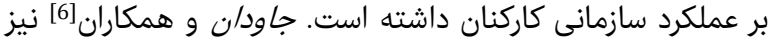

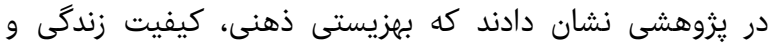
سلامت روانى با هم رابطه معنى داري دارني دارند.

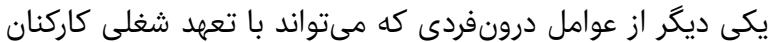

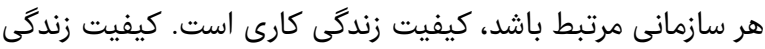

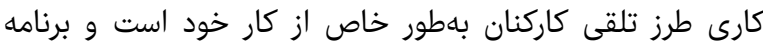

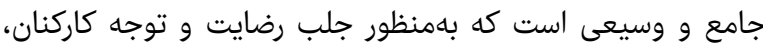

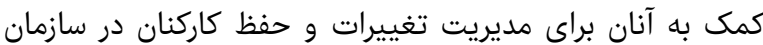

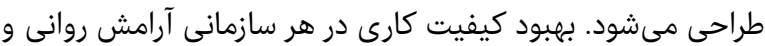

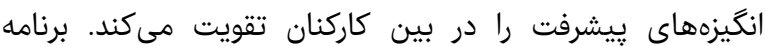

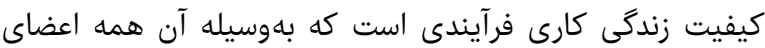

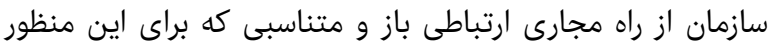

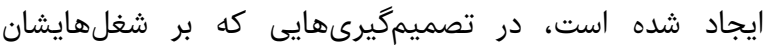

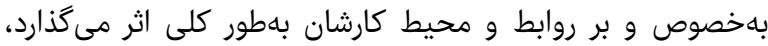

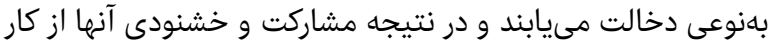

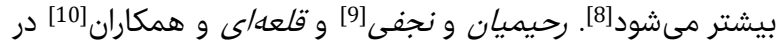

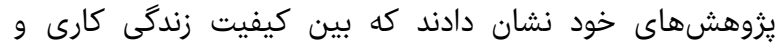

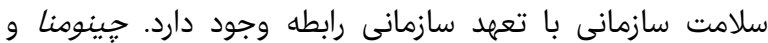

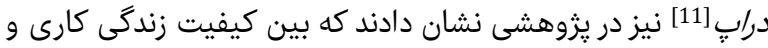

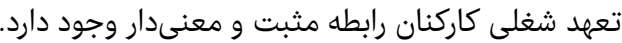

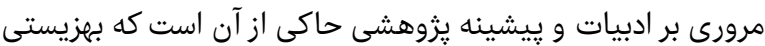

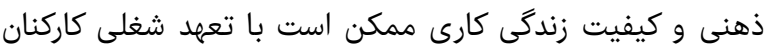

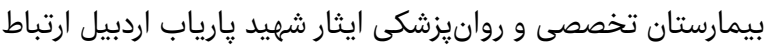

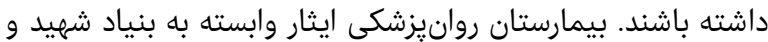

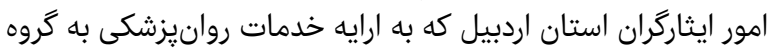

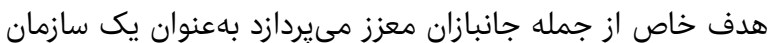

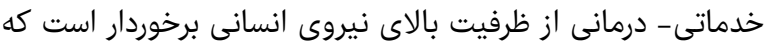

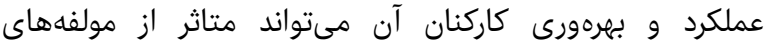

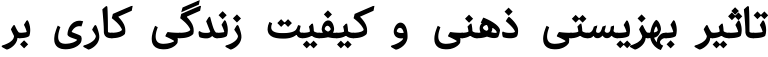 تعهد شغلى كاركنان بيمارستان}

MSc "على منصورلكورج

گروه مديريت، واحد اردبيل، دانشكاه علمى كاربردى مديريت صنعتى، اردبيل، ايران

PhD داود عبداللهى إنماعي، دران

كَروه علوم اجتماعى، دانشكده علوم انسانى، دانشكاه فرهنگَيان، اردبيل، ايران

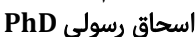

كروه مديريت، دانشكده علوم انسانى، واحد اردبيل، دانشگاه آزاد اسلامى، اردبيل،

جكيده

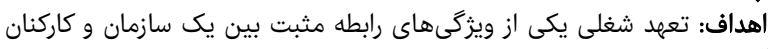

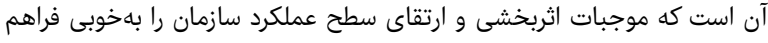

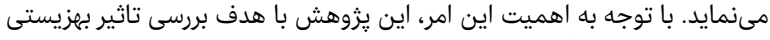

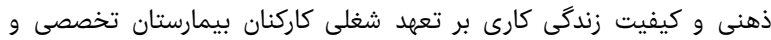

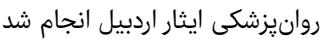

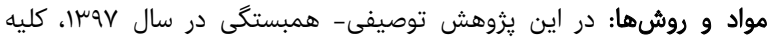

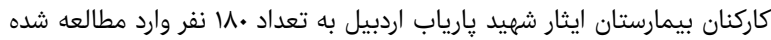

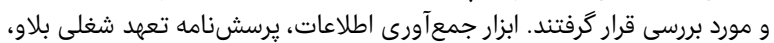

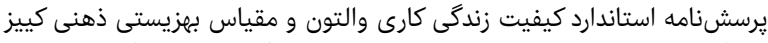

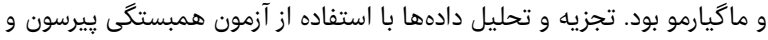

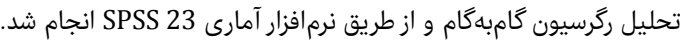

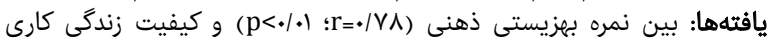

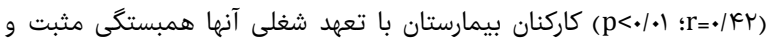

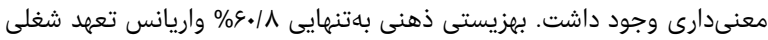

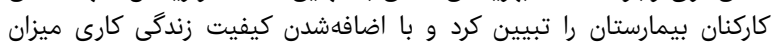

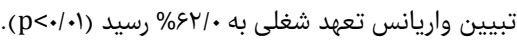

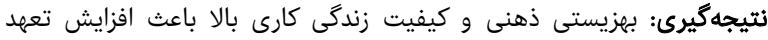

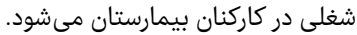

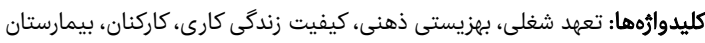

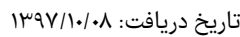

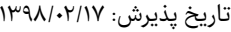

"نويسنده مسئول: تاريخ: amin.mansour1300@gmail.com

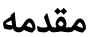

نيروى انسانى در هر سازمان از اركان اساسى آن سازمان به حساب

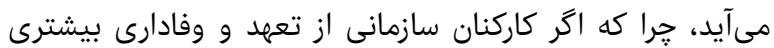

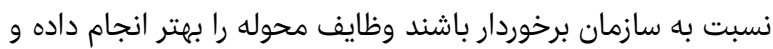

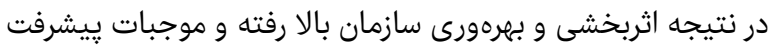

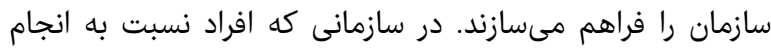

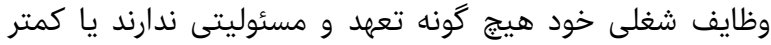

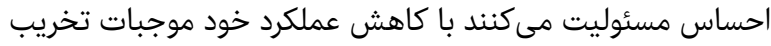

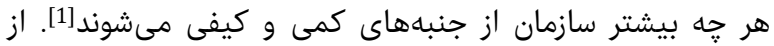

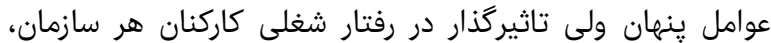

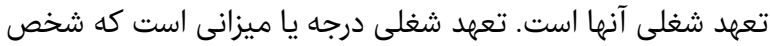

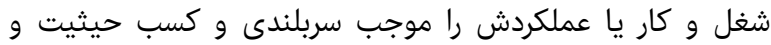

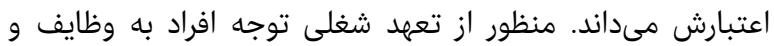

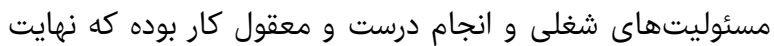

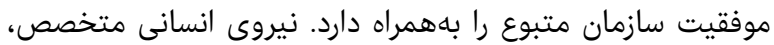

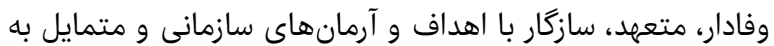

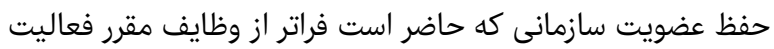

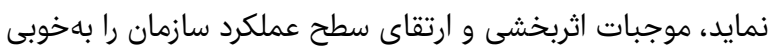

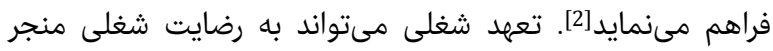
شود و رضايت از انجام كار موجب دلكرمى و ميل ميل بله ماندن درايت درلى منجر 


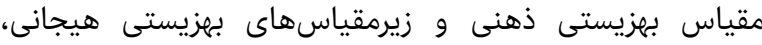

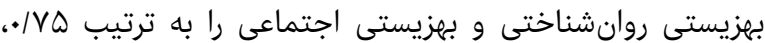

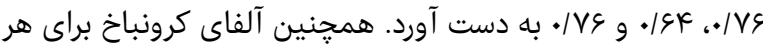

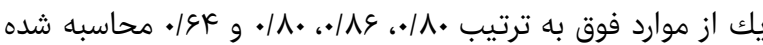

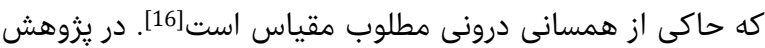

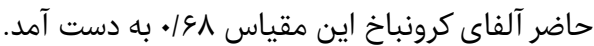

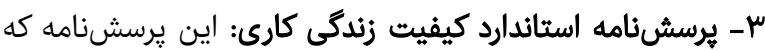

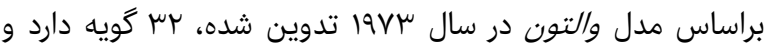

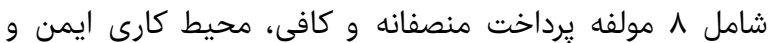

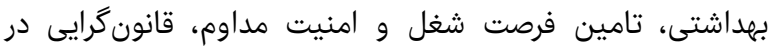

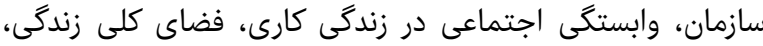

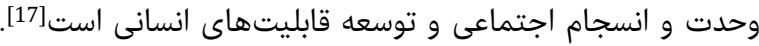

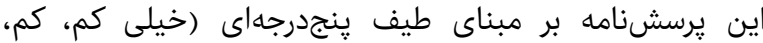

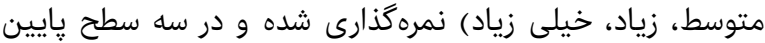

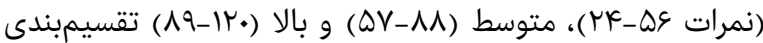

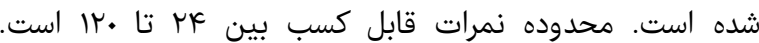

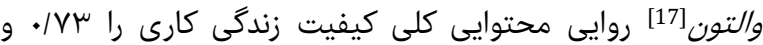

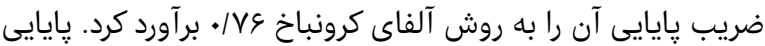

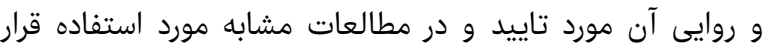

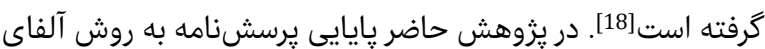

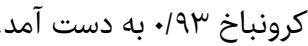

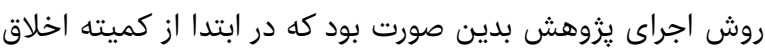

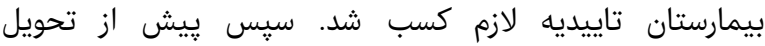

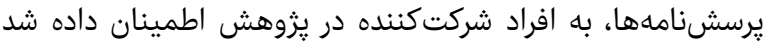

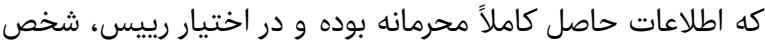

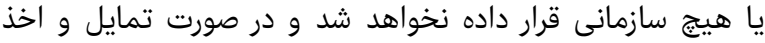

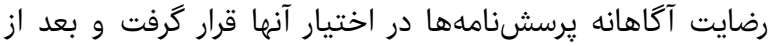

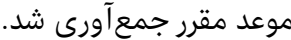

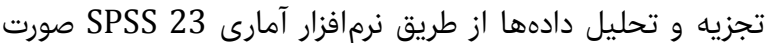

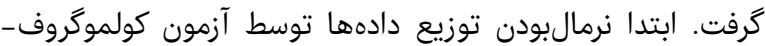

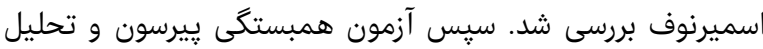

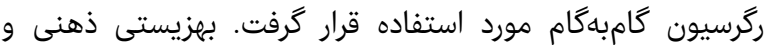

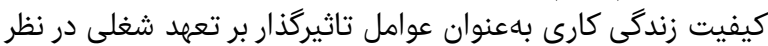

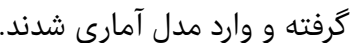

يافتهها

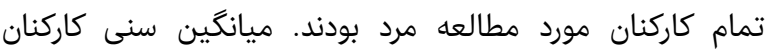
متاهl

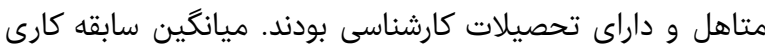

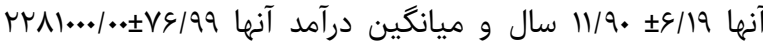

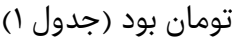

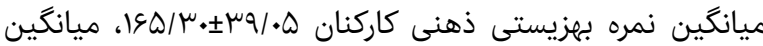

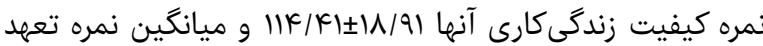

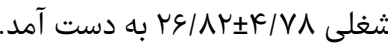

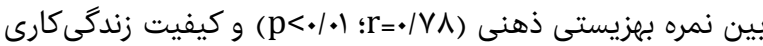

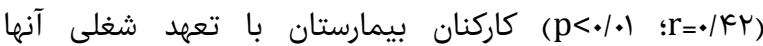

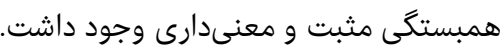

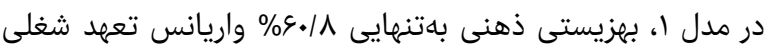

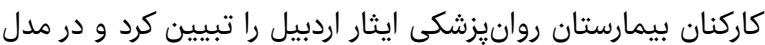

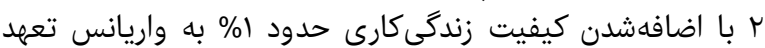

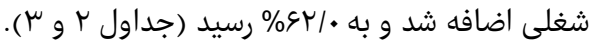

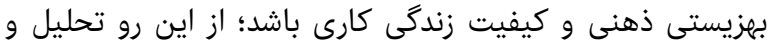

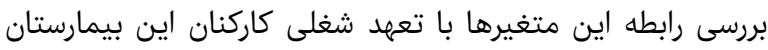

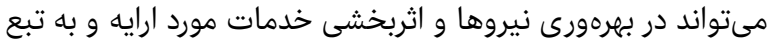

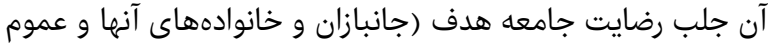
مردم) كارساز باشد.

بنابراين يزوهش كارساز باضد حاضر با هدف تعيين تاثير بهزيستى ذهنى و

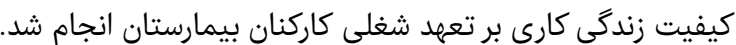

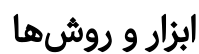

اين يزوهش يك روش مطالعه توصيفى- همبستخى است كه در در سال

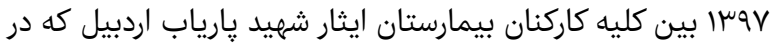

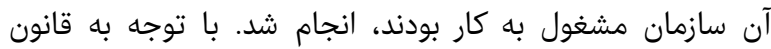

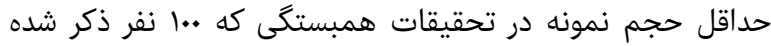

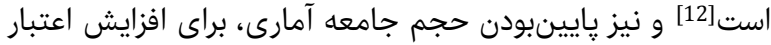

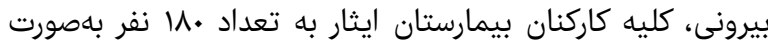

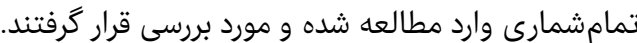

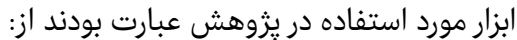

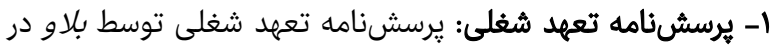

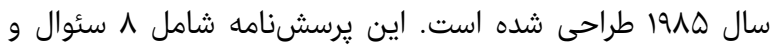

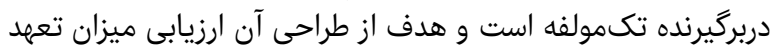

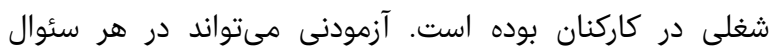

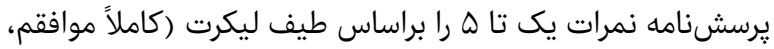

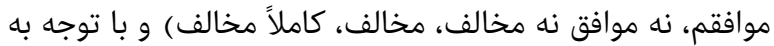

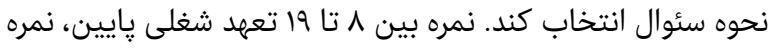

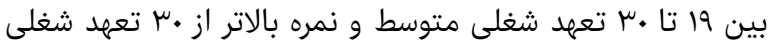

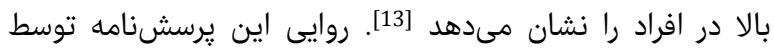

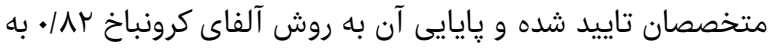

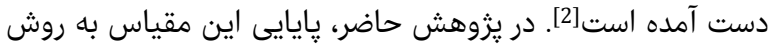
آلفاى كرونباخ ه 19. به دست آمده

r- مقياس بهزيستى ذهنى: اين مقياس توسط كييز و ماكيارومو [14]

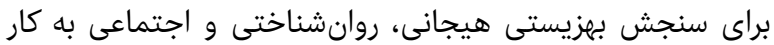

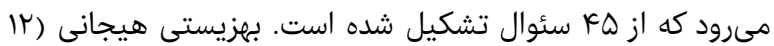

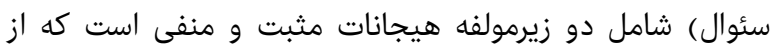

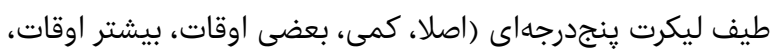

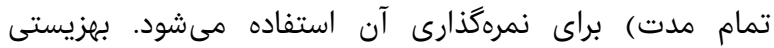

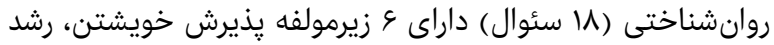

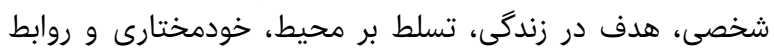

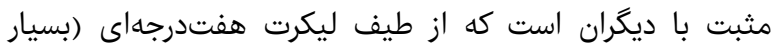

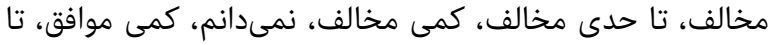

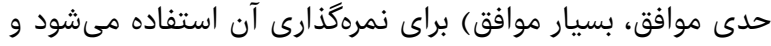

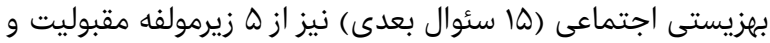

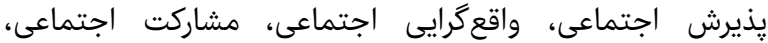

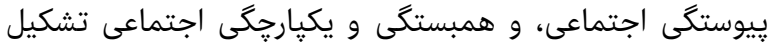

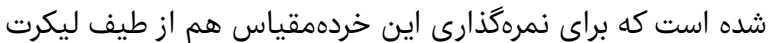
هفتدرجهاى (بسيار مخالف، تا حدى مخالف، كمى مخى مخالف،

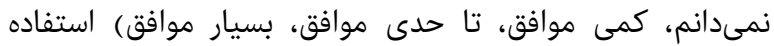

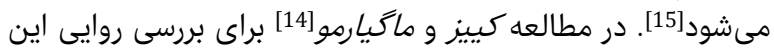

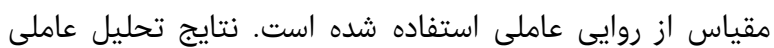

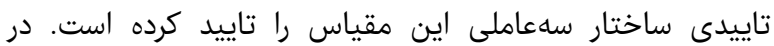

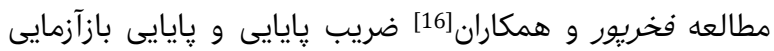

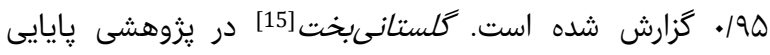


مطالعات غلامعلى لواسانى و همكاران[19]، گليرور و همكاران[20]

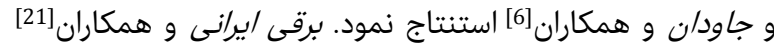

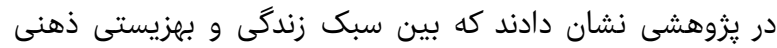

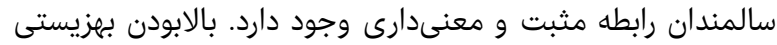

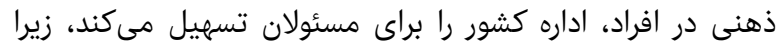

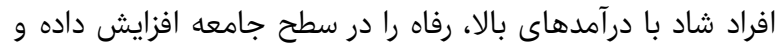

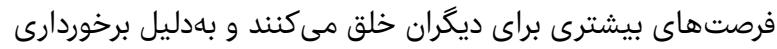

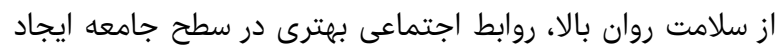

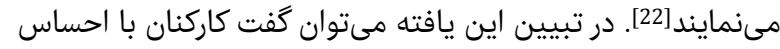

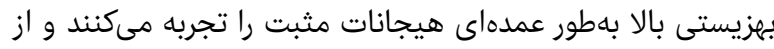

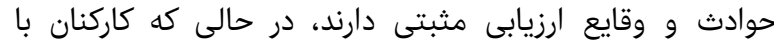

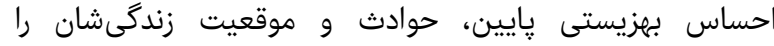

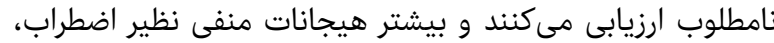

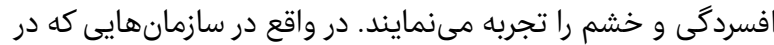

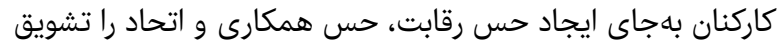

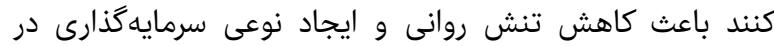

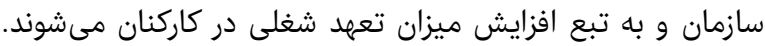

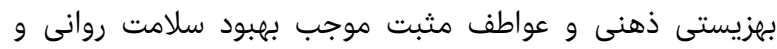

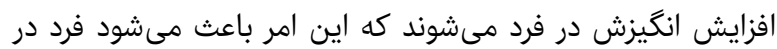

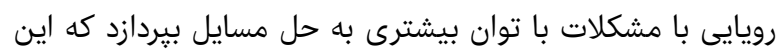

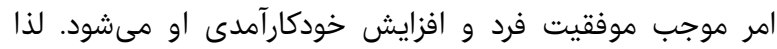

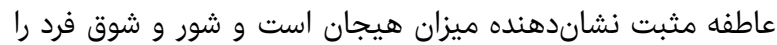

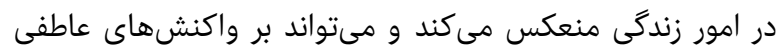

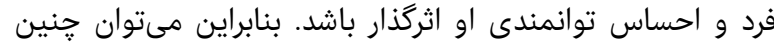

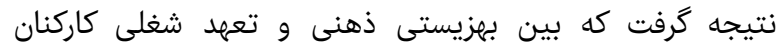

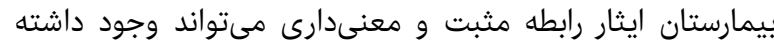
باشد.

بهزيستى ذهنى شامل اصول مهمى است كه از طريق تاثير بر

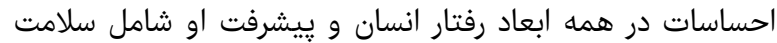

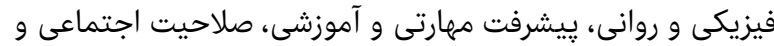

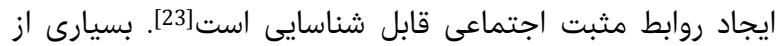

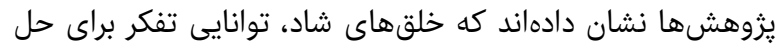

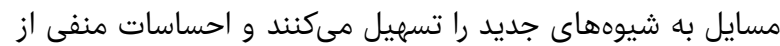

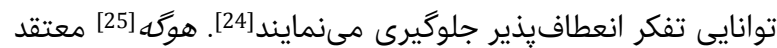

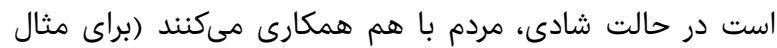

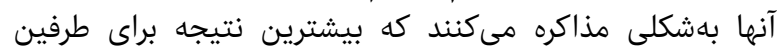

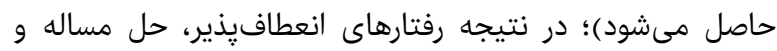

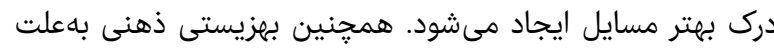

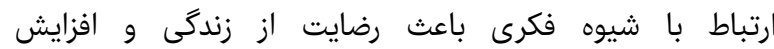

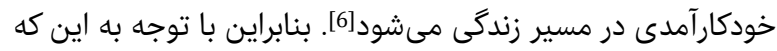

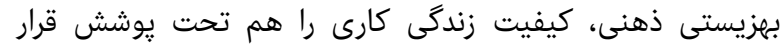

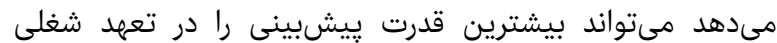

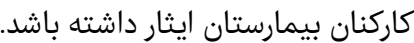

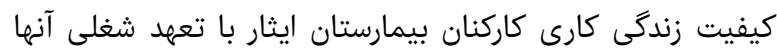

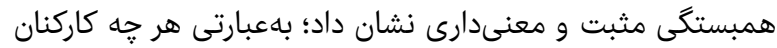

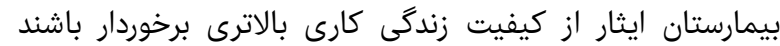

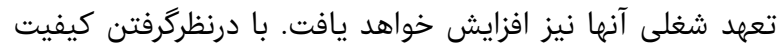

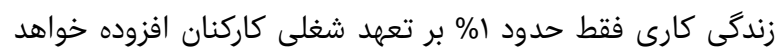
شد كه درصد قابل توجهى نئ نيست

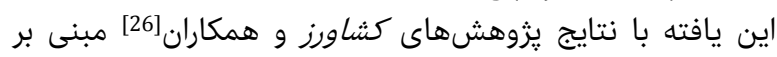

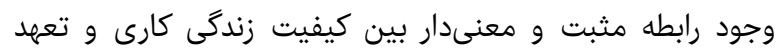

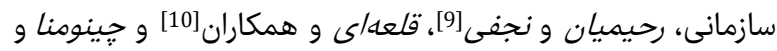

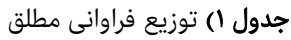

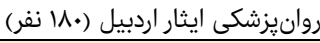

\begin{tabular}{|c|c|c|}
\hline درصد & تعداد & متغيرهاى دموگرافيك \\
\hline & & وضعيت تاهل \\
\hline $1 . \%$ & 14 & مجرد \\
\hline 9.1 & I\&Y & متاهل \\
\hline & & سطح تحصيلات \\
\hline V/r & Ir & سيكل \\
\hline$\| k / F$ & re & دييلم \\
\hline s/V & ir & فوقدييلم \\
\hline FA/ & $\wedge \vee$ & كارشناسى \\
\hline Q/द & 1. & كارشناسى ارشد \\
\hline $\mathrm{IV} / \Lambda$ & rr & دكترا \\
\hline & & دامنه سنى (سال) \\
\hline $11 / 1$ & $r \cdot$ & $r k-r A$ \\
\hline$M / \mu$ & س & $r q-\mu \mu$ \\
\hline$r \mu / q$ & kr & $\mu F_{-}{ }^{\prime} \alpha$ \\
\hline rN/Q & $\Delta r$ & $\mu q_{-} k \mu$ \\
\hline$I T / r$ & r & $k k_{-}-k \lambda$ \\
\hline$\mu / q$ & $\checkmark$ & $k q-\Delta \mu$ \\
\hline $1 / V$ & r & $\Delta F-\Delta \Lambda$ \\
\hline
\end{tabular}

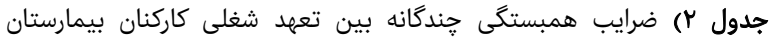

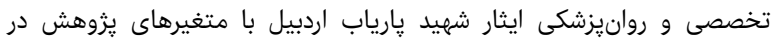

\begin{tabular}{|c|c|c|c|c|c|c|}
\hline معنى سطارى & خطاندار & F مقدار F & تعديل تريبن & ضعيين & همبستگ هند & كامبهكام الكوى \\
\hline $.1 . .1$ & $r / 99$ & $r V q / I \Delta K$ & $\cdot 19 \cdot 1$ & .19 .9 & $\cdot \mid$ VAI & مدل 1 \\
\hline.$/ .1$ & r/QF & SISkM & . & - $\mid$ SKK & ./Vq. & مدلץ \\
\hline
\end{tabular}

جدول س) ضرايب استاندارد و غيراستاندارد براى يِيشبينى تعهد شغلى براساس

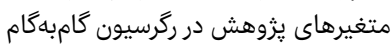

\begin{tabular}{|c|c|c|c|c|c|c|}
\hline معنى دارى & t مقدار & 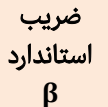 & خطاى اسارد & $\begin{array}{c}\text { غيراستاندارد } \\
\text { B }\end{array}$ & مدل & \\
\hline .1 .11 & $18 / V$. & ./VAI & $.1 . .9$ &.$/ .99$ & ذهزيستى & مدل \\
\hline. $\mid .1$ & $\mid \kappa / \Delta$. & . IVYq & .1 .09 & $.1 \cdot 19$ & ذهزيستى & مدل \\
\hline.$/ .11$ & $r / \Delta V$ & . & 更. & 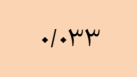 & زندگى كارى & $r$ \\
\hline
\end{tabular}

\section{بحثث}

يزوهش حاضر با هدف بروسى تاثير بهزيستى ذهنى و كيفيت

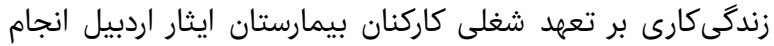

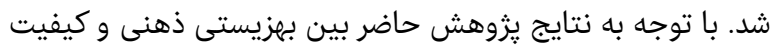

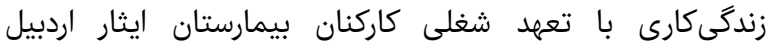

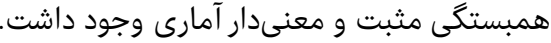

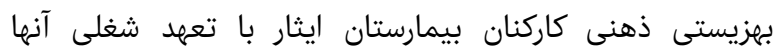

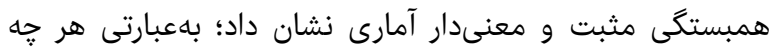

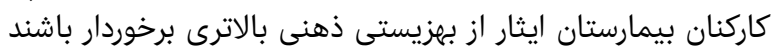

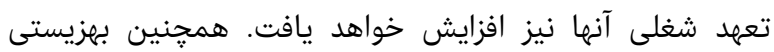

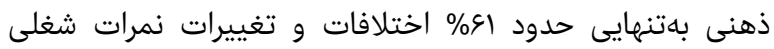

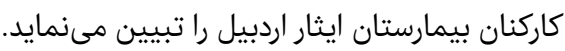

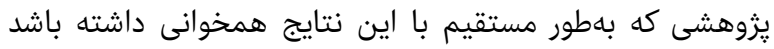

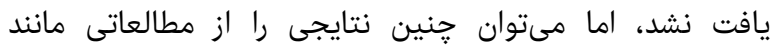


1- Jokar A, Afifian F. The relationship between job commitment and job satisfaction of employees working in libraries: A case study of Shiraz university. J Soc Sci. 2013;7(2):1-14. [Persian]

2- Banitaba M, Shahtalebi B, Karimi F. The study of job commitment and trust within the organization in high schools in Isfahan. J Instruction Eval. 2009;2(7):67-98. [Persian]

3- Kim WG, Brymer RA. The effects of ethical leadership on manager job satisfaction, commitment, behavioral outcomes, and firm performance. Int J Hosp Manag. 2011;30(4):1020-6.

4- Andam R, Montazeri A, Abavisani A. The relationship between work ethic, job commitment and job performance among employees of the offices of sport and youth in Khorasan Razavi Province. Contemp Stud Sport Manag. 2016;5(10):61-72. [Persian]

5- Ameri MH, Esmaeili M. The relationship among employees' empowerment, organizational commitment and job commitment in physical education organization of Tehran province. J Sport Manag. 2010;2(7):125-44. [Persian]

6- Javdan M, Haji Alizadeh K, Rafieipour A. The causal relationship between subjective well-being, quality of life and identity styles with academic achievement through academic self-efficacy in high school boy students in Bandar Abbas. Q J Res Educ Syst. 2015;9(30):123-45. [Persian]

7- Van De Voorde K, Paauwe J, Van Veldhoven M. Employee well-being and the HRM-organizational performance relationship: A review of quantitative studies. Int J Manag Rev. 2011;14(4):391-407.

8- Hadizadeh Talasaz Z, Nourani Sa'doddin Sh, Shakeri M T. Relationship between components of quality of work life with job satisfaction among midwives in Mashhad, 2014. Hayat. 2015;21(1):56-67. [Persian]

9- Rahimian H, Najafi A. An investigation of the relationship between quality of work Life and middle managers' performance in Iran national gas company. Q J Prod Manag. 2016;9(4):83-110. [Persian]

10- Ghaleei AR, Mohajeran B, Taajobi M, Imani B. Relationship between quality of work life and occupational stress in staff of Bu-ali Sina university of Hamadan, 2013. Pajouhan Sci J. 2015;13(4):60-6. [Persian]

11- Chinomona R, Dhurup R. The influence of quality of work life on employee job satisfaction, job commitment and tenure intention in the small and medium enterprise sector. South Afr J Econ Manag Sci. 2014;17(4):363-78.

12- Delavar A. Theoretical and practical research in the humanities and social sciences. 9th Edition. Teharn: Roshd Press; 2011. [Persian]

13- Mrayyan MT, Al-Faouri I. Nurses' career commitment and job performance: differences across hospitals. Nurs Leadersh (Tor Ont). 2008;21(2):e101-17.

14- Keyes CLM, Magyar-Moe JL. The measurement and utility of adult subjective well-being. In Lopez SJ, Snyder $\mathrm{CR}$, editors, Positive psychological assessment: A handbook of models and measures. Washington DC: American Psychological Association; 2003. pp. 411-25. 15- Golestanibakht T. Proposed model of subjective wellbeing and happiness in the population of Tehran [Dissertation]. Tehran: Alzahra University; 2007. [Persian]

16- Fakhrpour R, Yavari Y, Amirtash AM, Tondnevis F. Relationship between quality of work life and

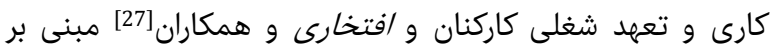

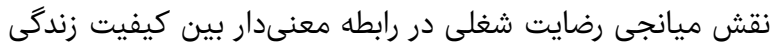

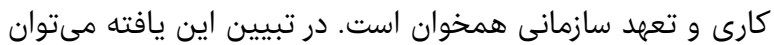

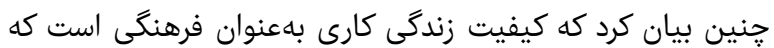

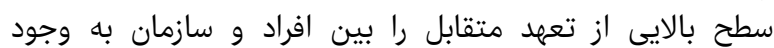

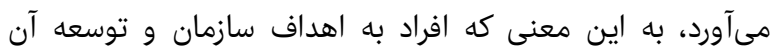

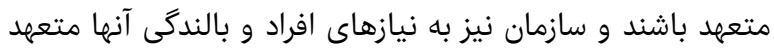

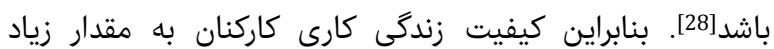

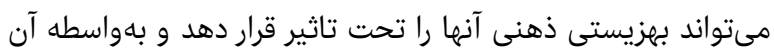

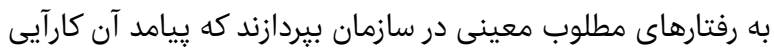

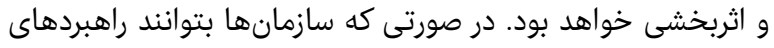

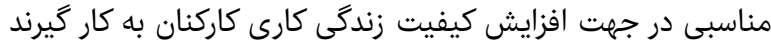

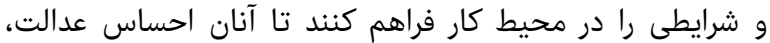

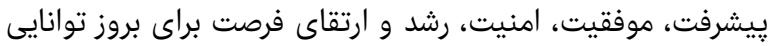

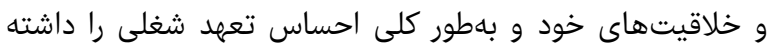

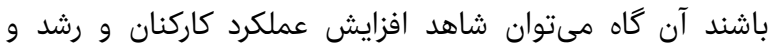

يويايى در سازمان بود [29].

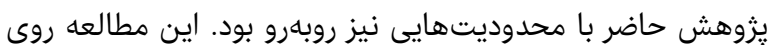

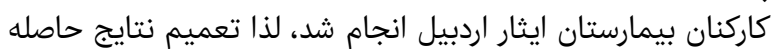

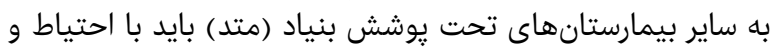

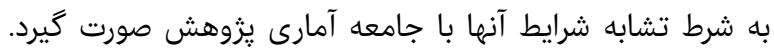

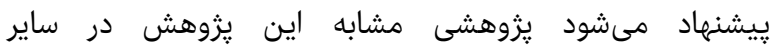

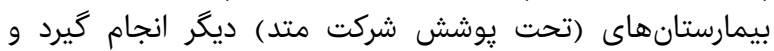

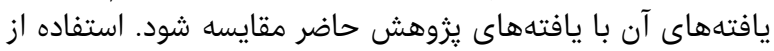

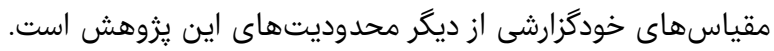

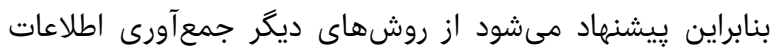

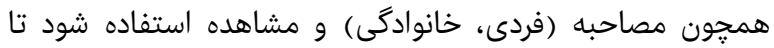

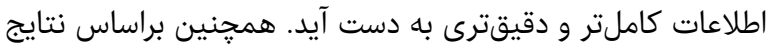

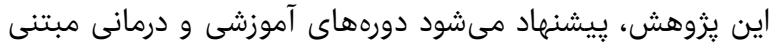

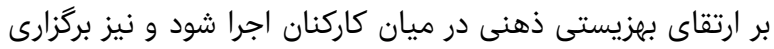

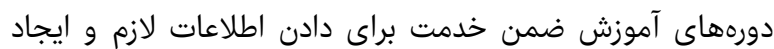

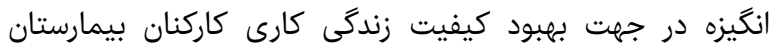
صورت گيرد.

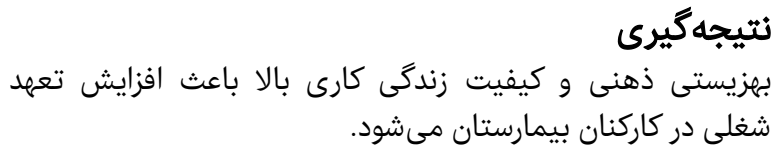

تشكر و قدردانى: نويسندگان اين مقاله از همكارى كاركنان

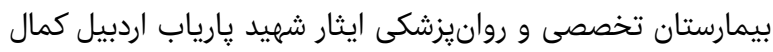
تشكر و قدردانى را به عمل مى تئي آورند.

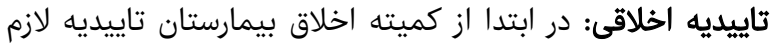

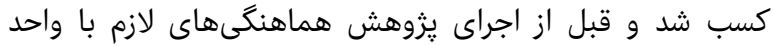

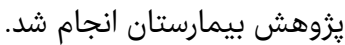

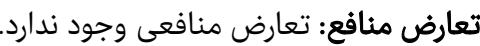

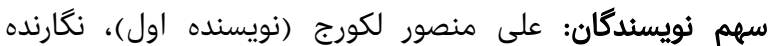

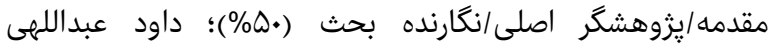

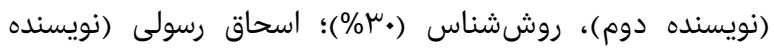
سوم)، تحليلكر آمارى (•r\%)

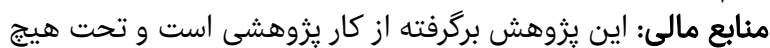

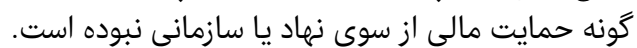

Volume 11, Issue 3, Summer 2019 
risk of hospitalization or an emergency room visit. Popul Health Manag. 2014;17(1):13-20.

24- Gasper K. When necessity is the mother of invention: Mood and problem solving. J Exp Soc Psychol. 2003;39(1):248-62.

25- Hooghe M. Is sexual well-being part of subjective well-being? An empirical analysis of Belgian (Flemish) survey data using an extended well-being scale. J Sex Res. 2012;49(2-3):264-73.

26- Keshavarz Mohammadi N, Zarei F, Rezaei M, Keshavarz A, Kalhor R. Exploring perspectives of medical staff on hospital's effects on their health: a health promoting hospital's approach. Razi J Med Sci. 2013;20(113):36-47. [Persian]

27- Eftekhari H, Bakhshayesh AR, Rahimi M. Modeling of the relationship between the quality of work life and organizational commitment by means of job satisfaction among elementary school teachers of Neyriz. J Mod Thoughts Educ. 2017;12(3):29-46. [Persian]

28- Thakre SB, Thakre SS, Thakre SN. Quality of work life of nurses working at tertiary health care institution: a cross sectional study. Int J Community Med Public Health. 2017;4(5):1627-36.

29- Kalhor M, Samiei Rad F, Garshasbi M, Mafi M, Ranjkesh F. Predictors of quality of work life and job performance in clinical staff in Qazvin, Iran. Int J Epidemiol Res. 2018;5(3):86-91.
IVF

organizational commitment in faculty members of physical education faculties and departments. J Sport Manag 2012;4(13):153-68. [Persian]

17- Walton RE. Quality of working Life: What Is It? Sloan Manag Rev. 1973;15(1):11-21.

18- Dehghan Nayeri N, Salehi T, Asadi Noghabi AA. Assessing the quality of work life, productivity of nurses and their relationship. Iran J Nurs Res. 2008;3(8-9):2737. [Persian]

19- Gholamali Lavasani M, Ejei J, Mohammadi Masiri F. Relationship between the meaning of life and optimism with subjective well-being. J Psychol. 2013;17(1):3-17. [Persian]

20- Golparvar M, Zeraati M, Salehi R. The relationship of work-family conflict and spillover to affective well-being of female nurses. Payavard. 2015;8(5):437-50. [Persian] 21- Barghi Irani Z, Rajabi M, Nazemi M, Bagiyan Koulemarz MJ. The role of lifestyle, psychological capital and self-care behaviors in predicting subjective wellbeing of diabetic elderly peoples. Health Psychol. 2017;6(23):17-38. [Persian]

22- Sarafino EP, Smith TW. Health psychology: Biopsychosocial interactions. $8^{\text {th }}$ Edition. Hoboken: John Wiley \& Sons; 2014.

23- Gandy WM, Coberley C, Pope JE, Rula EY. Well-being and employee health-How employees' well-Being scores interact with demographic factors to influence 